diagnosis and the condition found at operation. For this information I am indebted to those who kindly supplied me with particulars of the cases I have examined for them in my laboratory, and particularly to Mr. Mayo Robson, who has operated on many of the cases included in Groups 1, 2, and 4. The urines here dealt with form a consecutive series of 100 specimens, derived from 94 persons. All have been examined under the same conditions by the method I have described. From 29 specimens a more or less abundant deposit of crystals was obtained, but in 71 no crystalline deposit could be found even with the aid of the microscope. In 9 cases (forming Group 1) a stone was found in the pancreatic portion of the common duct at operation, and the pancreas was said to show evidence of disease. In 19 (Group 2), although no stone was found at the time of operation, there was said to be enlargement and hardening of the head of the pancreas. I found a gall stone in a specimen of faeces sent to me from one of these cases, and in another case included in this group I am informed that biliary calculi had been discovered in the stools shortly before I was asked to examine the urine. No reaction was obtained in 4 cases diagnosed as cancer of the pancreas (Group 3). One case in which the clinical symptoms and after-history of the case pointed to malignant disease of the pancreas yielded an abundant deposit. A preparation made from the urine of this case by the original $a$ method showed a mixture of fine crystals dissolving in 33 per cent. sulphuric acid in fifteen to twenty seconds, and many coarse, broad crystals that took five to six minutes to disappear. The urine was also found to contain a very large amount of bile and urobilin, and the excretion of chlorides was much diminished, while the urea and phosphates were approximately normal. In addition, the faeces when examined by the method I have recently described ${ }^{2}$ were found to be acid in reaction, to contain a large amount of undigested muscle fibre, and to yield not a trace of stercobilin. An estimation of the fats showed a total of 64.7 per cent., of which 56.3 per cent. was "neutral fat" and only 8.4 "fatty acid." So that the laboratory results, taken as a whole, confirmed the clinical diagnosis and pointed to cancer of the head of the pancreas, with complete obstruction of the common duct and a considerable degree of secondary inflammation. Of the remaining 67 specimens which gave no reaction, 9 were from cases of jaundice in which a calculus was found at operation in the gall bladder or in the common duct above the pancreatic portion (Group 4). In all these cases the pancreas was said to be normal in appearance and coneistency. Fifty-two were examined as controls. Of these, 30 were from patients suffering from diseases in which there was no reason to expect that the pancreas was involved (Group 5). They included cases of cancer of the stomach, colon, rectum, or liver, gastric ulcer, duodenal ulcer, gastritis, colitis, appendicitis, tuberculosis of the intestine, intestinal obstruction, cirrhosis of the liver, hepatic abscess, nephritis, floating kidney, tuberculosis of the kidney, cystitis, and Addison's disease. Twenty-two were from healthy individuals (Group 6). I have placed six cases in Group 7 which were under the care of Mr. Mayo Robson, and in which I had the opportunity of examining the urine one to two weeks after a cholecystenterostomy had been done for the relief of pancreatitis. All had given a well-marked reaction when the urine was examined shortly before the operation, and are included in Groups 1 and 2 , but when the second examination was made no reaction could be obtained.

I am not at present prepared to contend that at all times and under all conditions the results given by this improved method of examining the urine are indicative of the presence or absence of pancreatic inflammation, as my experience of it is not as yet sufficiently great, but I think that the evidence available suggests that it may be of considerable assistance in diagnosis, and that by means of this and other. methods of investigation I have described, the 50 per cent. of cases of pancreatic disease now said to be only recognized post mortem might be reduced to a much more creditable tigure.*

My experience suggests that it is always advisable to * Since writing this paper I have examined 93 specimens of urine most which were sent to me for the diagnosis of pancreatic disease, by this method, and in 54 , where the after-history has been obtainable, been confirmed. In the remaining 29 no reply has been received, or the case is too recent to allow of a definite opinion being formed. control the urine examination by an investigation of the faeces, for if the results agree, the chance of a mistaken opinion is considerably reduced and is probably small. The examination of the faeces is particularly useful in cancer cases, as the instance quoted in a former part of the paper clearly shows. It is of the utmost importance that malignant and inflammatory conditions of the pancreas should be clearly differentiated. Clinically it is often a matter of great difficulty; but if the results of the examination of the urine and faeces, carefully and conscientiously performed, are considered in conjunction with the history and symptoms, the chance of an erroneous diagnosis is materially reduced. I should, however, like to emphasize the fact that the laboratory investigations require a considerable amount of care, and that it is necessary to weigh all the evidence obtainable in forming a conclusion. The "pancreatic" reaction is only one factor that has to be taken into account in making a diagnosis in suspected cases of pancreatic disease or of jaundice in which it is sought to determine whether there is a gross obstruction to the free flow of bile into the intestine, and whether this obstruction when present is of a simple or malignant nature. 1 Lancet, March 19th, 1904. 2 BRITISH MEDICAL Journal, October 28th,

\section{EXTROVERSION OF THE BLADDER :}

Its: Treatment hy Extraperitoneal Implantation of the Ureters into the Rectum.

By C. J. BOND, F.R.C.S., SURGIEN TO THE LEICESTER INFIRMARY.

I wish to add another (hitherto unpublished) case of extraperitoneal rectal implantation of ureters for ectopia vesicae to the eight cases recorded by Dr. H. Simpson Newland in his interesting paper in the British MEdical JoURNaL of April 28th.

As Dr. Lendon says in his description of his original operation in an article in the same issue of the JourNal, it seems probable that extraperitoneal implantation of the ureters into the rectum will eventually become the method of the future in dealing with these distressing cases. And, indeed, any one who is familiar with the repeated operations, the imperfect immediate results, and the very unsatisfactory ultimate state of the patients when treated by the older plastic methods, will certainly endorse this opinion.

Having tried Maydl's intraperitoneal method unsuccessfully in a child of 8 weeks, in January, 1903, I operated on May 1st, 1903, on a healthy lad, aged 17, the subject of well-marked ectopia vesicae. After isolating each ureter with a small button of vesical mucous membrane, surrounding its orifice, as in Dr. Lendon's method, I separated the lower attachment of the vesical wall and inserted the mucous flaps containing the ureter endings into the wall of the rectum extraperitoneally by two separate incisions, one on either side of the gut. The lad is now, three years later, a fairly healthy young man of 20 . He has practically no difficulty with the urine, which is voided from the bowel four or five times in the 24 hours. Although his condition is so far satisfactory, there is no doubt that he passed, shortly after the operation, through a serious attack of pyelitis, first of one kidney and then of the other, and even now has occasional attacks of pain in the left kidney, with purulent urine.

It appears probable that every patient whose ureters are made to open into the colon or rectum undergoes some degree of ascending infection of those canals, and bearing in mind the presence of ascending currents in the ureters among other mucous canals, under certain conditions, as described in my Address on Surgery at the Leicester meeting of the Association last July, ${ }^{1}$ it is difficult to see how things could be otherwise. While under these new conditions the ureters must open into a septic cavity, yet the preservation of the normal valvular opening at the ureteral papillae, and hence the avoidance of any cicatrization of the outlet must be our chief safeguard in preventing stagnation of the contents of the ureter, the most potent contributory factor in the production of ascending infection. It will be interesting to learn whether the transplantation of the bladder wall and 
ureters intact, as described by $\mathrm{Mr}$. Moynihan in the Annals of Surgery for last montl, proves to be a further lielp in this direction.

There are other physiological points of much interest about these cases. It is well to realize the fact that $a$ patient whose ureters have been implanted into the rectum is practically receiving a rectal enema of two to three pints of urine daily, and it apparently takes some time for the bowel to become acclimatized to the new conditions, and to learn to expel, without absorption, the fluid poured into it. Our knowledge of the physiological effect of the absorption of urinary constituents through the intestinal mucous membrane is very scanty, but the peculiar attacks of malaise which my patient suffered from at times suggested the absorption of urine of a higher toxic character on such occasions.

There is also another point of interest concerning this function of urinary reservoir action acquired by the rectum. For the most part the urine is passed at lengthy intervals, $10 \mathrm{oz}$. to a pint at a time, clear and free from admixture with faeces, and this act is followed in a morning by the further evacuation, a little later, of a uormal firm motion without urine, while sometimes, in the early morning passage of urine, the first portion is more or less mixed with faeces. This phenomenon strongly confirms the suggestion that the rectum is habitually empty except shortly before the passage of a normal motion, and thus the urine collects in the recta anpulla free from faeces, is voided when the rectum becomes sufficiently distended, and that this act is followed, when necessity arises, by the descent into the rectum and the later evacuation of solid faeces. This habitual emptiness of the rectum further suggests this portion of the intestinal tube as a more suitable site for implantation than the colon higher up, which habitually contains faeces.

Finally, one word about the peculiarities of the vesical mucous membrane so extroverted. No attempt was made in my case to remove or cover in the exposed vesical mucous membrane, and yet, owing to the change in environment, the exposed surface gradually changed its eharacter and became a non-secreting skin surface, having all the appearance of cicatricial skin; this occurred all over, except at the angle formed by the abdominal wall and the epispadic penis, where a certain amount of moisture and mucus still collected, and here the epithelium preserved its mucoid type. In the language of heredity, the vesical, and probably the genito-urinary micous membrane, possesses the inborn capacity of developing along two lines-the mucous membrane and the skin-while the environment determines the course development shall take in any case at any given time, the practical point being that it is not necessary to remove such portions of exposed membrane when the normal secretion bathing the surface has been arrested.

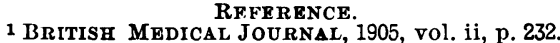

\section{TONSILLITIS IN CONVALESCENCE FROM DIPHTHERIA.}

By J. D. ROLLESTON, M.A., M.D.Oxon.,

ASSISTANT MEDICAL OFFICER AT THE GROVE FEVER HOSPITAL OF THE METROPOLITAN ASYLUMS BOARD.

THE occurrence of a non-specific inflammation of the tonsils in convalescence from diphtheria is not uncommon, and is of no little interest and importance. The concurrent symptoms suggest of ten in such cases the possibility of scarlet fever. On the other hand, a relapse of the disease may be suspected, and the advisability of serum treatment will at once have to be discussed. The following observations are based on observations made on 900 consecutive cases of diphtheria under my care during three years.

Apart from cases of inflammation of the tonsils which proved at the time or subsequently to be scarlatinal in origin, the tonsillitis occurred in 64 cases ( 7.1 per cent.) either within and usually towards the end of the first three weeks of the disease, or at a much later period. In the first class the tonsillitis found in 30 cases (3.3 per cent.) was invariably associated with late manifestations of serum, which consist in a rash generally of the type of a circinate erythema, and usually associated with pyrexia, less frequently with pains in the joints or muscles, and adenitis of the submaxillary and cervical glands. This faucial affection, described by the French under the name of pseudo-relapse, or angine de retour, ${ }^{1}$ may be conveniently designated angina redux. It occurs, as a rule, within a fortnight from the first injection of antitoxin, the average time in these 30 cases being 14.1 days after the first injection. Similar figures are given by sevestre and Aubertin, ${ }^{2}$ who mention the reappearance of angina and croup in the period of late serum phenomena on the tenth to the fifteenth day after injection.

The appearances presented by the throat in this condition vary from an injection of the fauces with a follicular deposit on the tonsils to large semi-confluent patches resembling the early stage of diplitheria. Unlike diphtheria, however, they are not adherent, and disappear often without any local treatment whatsoever as the other serum phenomena subside, leaving no ulceration. In only one of my cases was there any loss of tissue in the subjacent mucosa.

Bacteriological examination reveals rich growths of staphylococci and streptococci, which accords with the theory that the late manifestations of serum are due to the action of these micro-organisms. ${ }^{3}$ As a rule the patients do not complain of sore throat. Albuminuria may develop at this time, or pre-existing albumen in the urine may increase in amount. Pyrexia may persist for several days, and the constitutional disturbance, especially in older patients, be considerable. The average duration of the pyrexia was 5.2 days. In 2 cases the temperature reached $104^{\circ}$; in 5 it exceeded $103^{\circ}$.

Submaxillary and cervical adenitis is relatively so common at this period ${ }^{4}$ without any striking corresponding inflammation of the throat, that an inspection of the fauces in such cases is often omitted. The absence of a more general recognition of angina redux is thus ex. plained. This condition, though by no means common, is more frequent than relapses of diphtheria, the existence of which is not disputed. Relapses occurred twelve times- that is, 1.3 per cent,-in my 900 cases.

The statistics ${ }^{5}$ of the Metropolitan Asylums Board hospitals during the last six years give similar figures-1.16 to 2.11 per cent., or an average of 1.61. The occurrence of laryngeal symptoms at this period has been recorded by Sevestre and Aubertin, ${ }^{4}$ in some cases sufficiently severe to necessitate intubation. Such phenomena, which have been attributed to laryngeal oedema produced by the serum, I have never witnessed myself.

Secondary angina in convalescence from diphtheria, apart from serum manifestations, is not uncommon. It occurred in 34 of my cases ( 3.7 per cent.), the average time being the fortieth day. It is thus seen to be of more common occurrence than relapses, nor is the reason of this far to seek. Whereas the faucial tissues have gained a certain degree of immunity against the specific infection, their power of resistance against other forms of inflammation has been lowered.

A precisely similar event occurs in convalescence from scarlet fever where tonsillitis is a well-recognized sequela, occurring in 1.73 to 3.32 per cent., or an average of 2.44 per cent. for the last six years in the Asylums Board hospitals, as compared with the frequency of relapses, ${ }^{*}$ which varied from 1.09 to 2.01 per cent. during the same period. ${ }^{5}$

Like tonsillitis in convalescence from scarlet fever, the late tonsillitis of diphtheria occurs more frequently in adults and older children, in females and in the damp months of the year. Thus, in 34 cases 11 were above 7 years of age, 16 were males, 18 were females. Seven of the cases occurred in January, 3 in each of the months of February, March, and April ; 2 in each of the months of August, September, and October; and 12 in November alone. The accumulation of acute cases in the same ward was not found to have any influence in the causation of this late tonsillitis, as it is said to have in the case of late scarlatinal tonsillitis. It is possible, however, that the condition may be more common in hospital than in private practice.

The symptoms were slight, as a rule. In two cases vomiting first drew attention to the condition. The constitutional disturbance was not so great, nor the temperature so high, as in angina redux. The average duration * It is more than probable that a large percentage of so-called relapses
of scarlet fever are in reality primary attacks. 